\title{
Particle-image velocimetry measurement errors when imaging through a transparent engine cylinder
}

\author{
David L Reuss ${ }^{1,4}$, Marcus Megerle ${ }^{2}$ and Volker Sick ${ }^{3}$ \\ ${ }^{1}$ General Motors R \& D Center, 30500 Mound Road, Mail Code 480-106-252, Warren, \\ MI 48090-9055, USA \\ ${ }^{2}$ Sandia National Laboratories, Combustion Research Facility, MS-9053, PO Box 969, \\ Livermore, CA 94551-0969, USA \\ ${ }^{3}$ Department of Mechanical Engineering, University of Michigan, 2202 GG Brown Building, \\ 2350 Hayward Street, Ann Arbor, MI 48109-2125, USA \\ E-mail: david.1.reuss@gm.com,mvmeger@ca.sandia.gov and vsick@umich.edu
}

Received 4 December 2001, in final form 3 April 2002, accepted for publication 16 April 2002

Published 20 June 2002

Online at stacks.iop.org/MST/13/1029

\begin{abstract}
When making particle-image velocimetry measurements through the quartz cylinder of a reciprocating engine, the particle images are aberrated. This work quantifies the practical field-of-view and the errors in the velocity measurements caused by those aberrations. Electro-optical image shifting was used to create a repeatable particle-image displacement distribution for 60 images. Ensemble averaging of these images is used to quantify the rms errors due to the shot-to-shot variation in (1) the particle-image fields,

(2) the camera noise, (3) the variance in the correlation-peak detection and

(4) the particle-image aberrations. These results demonstrate that the

field-of-view is restricted to the centre $66 \mathrm{~mm}$ of the $86 \mathrm{~mm}$ inside-diameter cylinder due to decreased accuracy, decreased image-to-image precision and decreased displacement-peak detectability of the image-displacement correlation. The correlation-peak detectability was degraded by both particle-image aberrations and decreased transmission of the scattered light.
\end{abstract}

Keywords: digital particle image velocimetry, measurement precision

(Some figures in this article are in colour only in the electronic version)

\section{Introduction}

Particle-image velocimetry (PIV) measurements in a reciprocating internal combustion (RIC) engine cylinder require imaging through a thick-walled, transparent, cylindrical cylinder to capture the velocity distributions of the tumble planes (planes parallel to the cylinder axis). It is desirable to create neardiffraction-limited particle images that are two to three times the pixel size at the image plane. Imaging through the cylinder causes aberrations, and thus diffraction-limited imaging is not possible. As described by Reeves (1995), the dominant aberrations are for particles that are near the cylinder wall in

4 Author to whom any correspondece should be addressed. a plane normal to the optical axis. The cylinder aberrations cause

(1) nonlinear changes to the image-plane coordinate system,

(2) asymmetric particle-image distortions, and

(3) increased particle-image size.

These aberrations result from the fact that the meridional and sagittal scattered-light rays follow different optical paths through the cylinder, as shown in figure 1; consequently, meridional and sagittal rays from off-axis particles focus at different $z$ positions. At a $z$ position between the meridional and sagittal focal positions lies the circle of least confusion, which is the position where the images appear most round. In practice, the position of the circle of least confusion would 

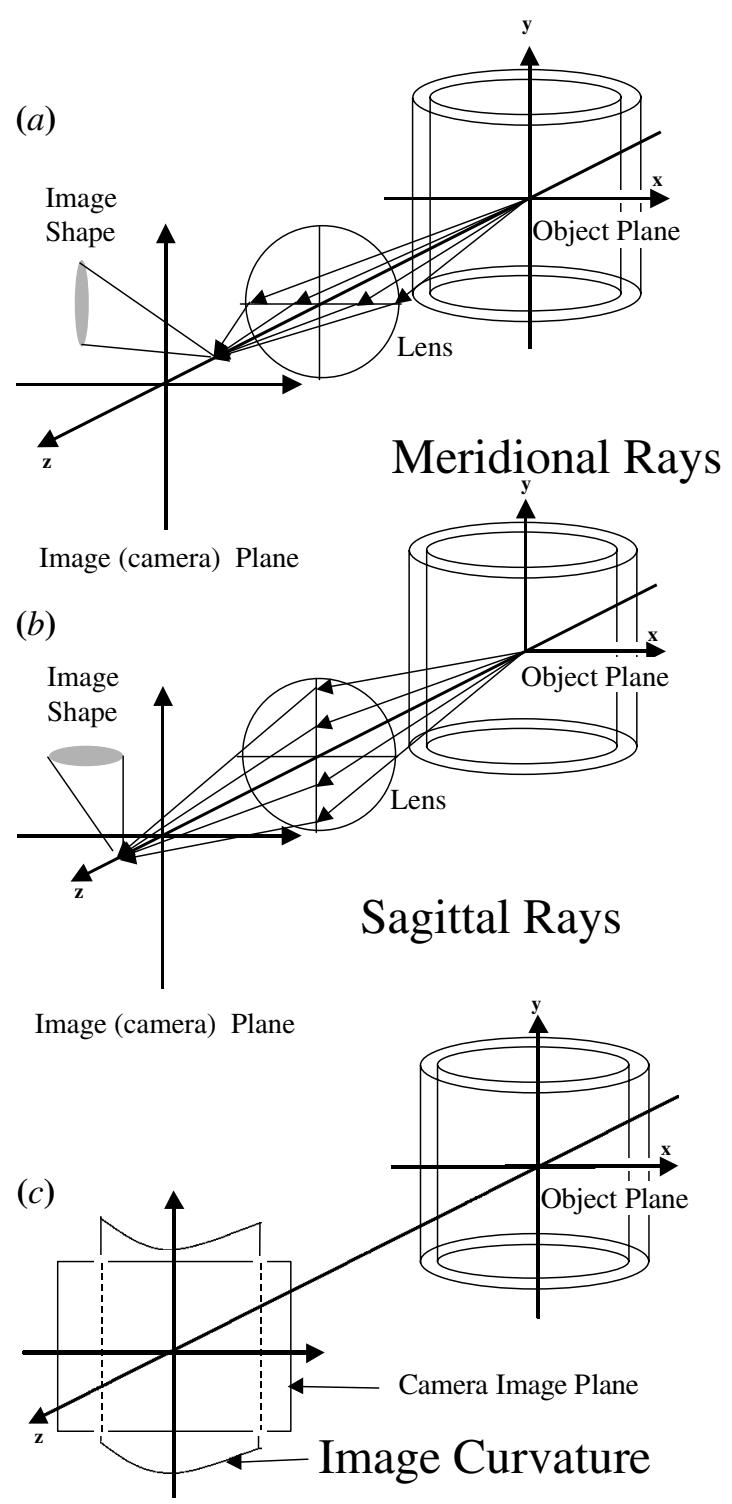

Figure 1. Illustration of meridional and sagittal rays.

be identified as the best focal position. With no correction all three image planes curve, as indicated in figure 1(c). There is a fourth problem caused by the cylinder due to inadequate light transmission. As predicted by Fresnel's equations (Fowels 1975), the amount of reflected and transmitted light at each interface is a function of internal or external reflection, angle of incidence, and polarization. As a consequence, for increasing $x$ a position is reached where the intensity of the images is too small to be detected. This will be shown to result in a practical limit to the field of view as well, irrespective of the aberration problems.

It is possible to correct for the aberrations noted above as described by Reeves (1995). This is especially important for photographic recording where near-unity magnification is used. However, with digital recording, where large demagnification is used, the effects may be less and it is of value to determine the severity of the problems when imaging without correction. The purpose of this work is to quantify (1) the practical field-of-view and (2) the errors in the velocity measurements due to the aberrations caused by the cylinder when imaging without a correction lens. The methodology used here is identical to that used by Megerle $e$ al (2002), but imaging through a cylinder rather than a thin, flat window. To quantify the error, electro-optical image shifting is used to create a precisely repeatable image displacement over the camera image, and thereby simulate a repeatable velocity distribution (Landreth and Adrian 1988). The particle-image displacement error is quantified by determining the ensemble-average and rms particle-image displacement (Reuss 1993). Also, the technique quantifies the correlationpeak detection losses caused by both the increased particleimage aberration and decreased light transmission near the cylinder walls. Digital PIV with double-exposure singleimage cross-correlation is used to measure the particle-image displacements. Although double-image cross-correlation is ultimately a better solution, these results are applicable when double-exposure photographic recording is used (Reuss 2000). Also, the single-image recording used in this study provided zero particle displacements, thereby creating particle-image displacements that are the result of only the electro-optical image shifting.

The experimental method described above measures the systematic error in the ensemble-average displacement distribution caused by cylinder aberration of the particle images. This technique does not quantify nonlinear coordinate distortion, which can be corrected by coordinate correction of the recorded images. The rms noise about this ensemble average distribution includes

(1) the error due to the random particle fields,

(2) the image-to-image displacement error due to the digital PIV detection algorithm, and

(3) the effect of the systematic, cylinder-induced aberration of the particle images on both (1) and (2).

This technique does not include increased noise and decreased correlation-peak detection due to velocity gradients and outof-plane pairing losses. Finally, due to the laser illumination configuration, these experiments do not include the kind of background noise experienced in operational engine experiments.

\section{Experiment}

The imaging work was conducted in a fused silica cylinder with inside and outside diameters of 86 and $113 \mathrm{~mm}$, respectively. This is representative of a 0.51 single-cylinder engine. The ends of the tube were sealed with flat windows.

A TSI 9306 six-jet atomizer was used to generate the $1 \mu \mathrm{m}$ silicone-oil droplets. This atomizer/oil system has been used in engine experiments and the $1 \mu \mathrm{m}$ size verified with laser Doppler anemometry. The cylinder was flooded with a burst of an air jet laden with droplets and homogenized by pulling a perforated plate through the tube. The plate had an $85 \mathrm{~mm}$ diameter and was perforated with a matrix of $6 \mathrm{~mm}$ holes. The particles were allowed to settle but would remain suspended for about $15 \mathrm{~min}$, the number density decreasing with time. Oil deposition on the walls was insignificant during the tests. Estimates of the number density and homogeneity were made by counting the number of particle images in $32 \times 32$ pixel 


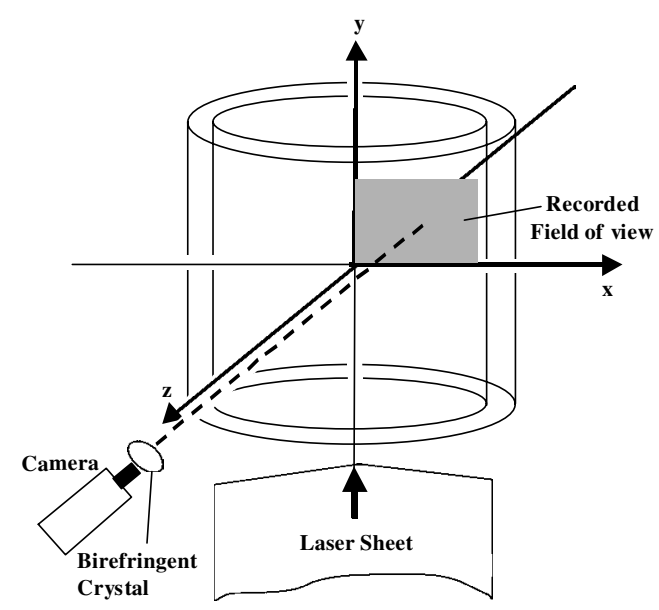

Figure 2. Illustration of the experimental configuration.

regions. The particle images were, of course, larger than expected from the magnification due to the finite aperture and the aberrations imposed by the quartz cylinder.

The camera was a LaVision FlowMaster 3S, which has a $1024 \times 1280 \mathrm{CCD}$ sensor of $6.7 \times 6.7 \mu \mathrm{m}$ pixels, $12 \mathrm{bit}$ dynamic range with $<2$ counts rms noise. The camera was fitted with a $105 \mathrm{~mm}$ Nikon MicroNikkor ED lens, set at an aperture of $f / 4$ and 0.214 magnification for particle imaging. The magnification was adjusted by imaging on a known grid in the absence of the cylinder, the lens focusing adjustment thereafter held fixed. The camera was then translated while observing the images to obtain the position of best focus. The field of view was $x, y=40 \mathrm{~mm} \times 32 \mathrm{~mm}$, offset from the centre as illustrated in figure 2. The particle images were resolved by 3 or more pixels.

A dual-resonator, $532 \mathrm{~nm}$ wavelength, Nd:YAG laser was used to illuminate the particles. The two, coincident, $0.5 \mathrm{~mm}$ thick laser sheets were cross-polarized with their polarization axes in the $y$ and $z$ directions. The $10 \mathrm{~ns}$ laser pulses were triggered simultaneously. The coincident laser sheets passed through the ends of the cylinder, thereby illuminating a $x-y$ plane for the desired values of $z$ (refer to figure 2). Allowing the laser sheet to enter and exit the tube longitudinally eliminates scattering from the tube surfaces.

Electro-optical image shifting is a means of creating a nearly constant image displacement over the field of view that is identical from image to image. Theoretical discussion of electro-optical image shifting can be found in Landreth and Adrian (1988) and its application is described in Reuss (1993). In short, electro-optical image shifting creates a repeatable particle-image displacement distribution as follows. The particles are illuminated with cross-polarized laser sheets and imaged through a properly oriented birefringent uniaxial crystal plate. In this way a stationary particle results in two images separated by the imposed shift. The electrooptical image displacement distribution does have a systematic variation that is a function of $x$ and $y$, but is the same in each exposure at any given $x, y$ position. In this study the systematic spatial variation was $<1 \%$. Particles in a flow with a finite laser-pulse separation create two images whose separation is the sum of the electro-optical image shift plus the velocity-imposed displacement (refer to Reuss (1993)).
This was originally developed by Landreth and Adrian (1988) for removing the directional ambiguity inherent in doubleexposure photographic PIV. Implementation in this study was accomplished by placing a $3 \mathrm{~mm}$ thick birefringent crystal just ahead of the camera lens. The cross-polarized laser pulses in this study were simultaneous and thus the particles experienced no flow-induced displacement. Therefore, the recorded particle-image displacement distribution was due to the birefringent crystal alone. In this study, a shift of approximately 8.7 pixels in the $x$ direction and a 0.6 pixel shift in the $y$ direction were measured for the magnification of 0.214. The difference from CCD image to CCD image is that different particles are present in the laser sheets due to the approximately $1 \mathrm{~cm} \mathrm{~s}^{-1}$ residual convection; however, the image shift distribution is the same.

The double-exposure images were processed with LaVision PIV Interrogation software, version 6.04, using what is referred to as the 'auto-correlation function with offset'. This is actually the cross-correlation of the $32 \times 32$ pixel region at a grid node with a different $32 \times 32$ region, which was offset by an 8 pixel shift in the direction of the image displacement. The $32 \times 32$ interrogation area contained 5-10 particle pairs. No postinterrogation processing was used.

The effect of the cylinder on the displacement accuracy is determined by measuring the change in the ensembleaverage displacement with and without the cylinder, where the ensemble average is defined by

$$
\langle\delta x\rangle_{i j}=\frac{1}{N} \sum_{n} \delta x_{i j}
$$

and $n$ is the image number. The ensemble-average displacement distribution was computed for 60 CCD images, recorded and interrogated at each $z$ position. The precision of the measurements is quantified by the rms variation about the ensemble average at each interrogation node for the 60 samples at each node, namely

$$
\operatorname{rms} \delta x_{i j}=\left(\frac{1}{N} \sum_{n}\left(\delta x_{i j}-\langle\delta x\rangle_{i j}\right)^{2}\right)^{1 / 2}
$$

where $i$ and $j$ are the grid indices. The systematic variation of the displacement distribution does not contribute to the rms noise computed in this study since the variation is computed about the average value at each node.

\section{Results}

\subsection{Focal planes}

The first tests were to determine the positions of the three focal planes (meridional focus, sagittal focus and circle of least confusion (CLC)). They were measured across the field of view in the $x$ direction and in different planes in the $z$ direction. The focal positions were determined by two different techniques. In the first, an USAF Resolution Target was placed at the position of interest, back-illuminated with a diffuse, incoherent (green-filtered white light) light source, and imaged with the PIV camera. These results are shown only to illustrate the curvature of the CLC plane. The camera lens was set for fixed magnification, $M=0.107$, and moved with a translation stage 
- Meridional Focus

- CLC, Particle

$\triangle$ Sagittal Focus

- CLC, USAF TArget

Cylinder Inside Radius

$\mathbf{X}, \mathbf{m m}$

$\begin{array}{llllllllll}0 & 5 & 10 & 15 & 20 & 25 & 30 & 35 & 40 & 45\end{array}$

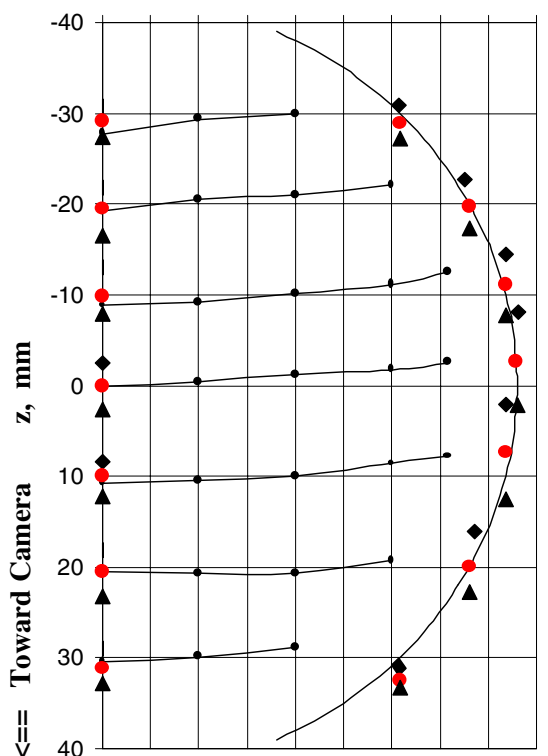

Figure 3. Image planes as viewed from large $y$ looking toward $y=0$. In this plot $z=0$ indicates the camera is positioned at the best-focus-image plane corresponding to the object plane at $z=0$.

to determine the CLC. This was presumed to be at the position where the horizontal and vertical bars of the resolution target were equally resolved. This determination was repeated with the USAF target at object planes of $z=0, \pm 10, \pm 20$ and $\pm 28 \mathrm{~mm}$. The curvature of the CLC planes are shown as full curves in figure 3 (careful comparison with figure 1 is suggested to orient the perspective in figure 3 ).

The second technique was to identify all three focal planes using the $1 \mu \mathrm{m}$ oil droplets illuminated with the laser sheet and imaged at $x=0$ and for $x$ as near the wall as possible. Again the camera was translated with fixed magnification, but at $M=$ 0.214 . This determination was repeated with the laser sheet at $z=0, \pm 10, \pm 20$ and $\pm 30 \mathrm{~mm}$ (rather than 28 for the USAF Resolution Target). To change the $z$ position, the laser sheet was held fixed, the cylinder moved to the $z$ position of interest, and the camera then moved to find the corresponding focal plane. The camera position was referenced to zero by focusing at the CLC for $x, y, z=0,0,0$. The results in figure 3 reveal the field curvature and the extreme separation of the meridional and sagittal foci (up to $8 \mathrm{~mm}$ ) near the cylinder wall (large $x$ ). A comparison of figures 1 and 3 is useful to orient the relative positions of the three focal planes. It can be seen that object planes near the centre of the cylinder $(z=-10,0$, and +10$)$ show the most curvature and largest meridional-to-sagittal separation. Figure 3 also demonstrates that, in comparison with the meridional rays, the sagittal rays focus nearest the flat image plane. As suggested by Reeves, this demonstrates that correcting the meridional rays alone will do the most to flatten the field by moving the meridional focal positions (and therefore the CLC) toward the unaberrated focal plane.

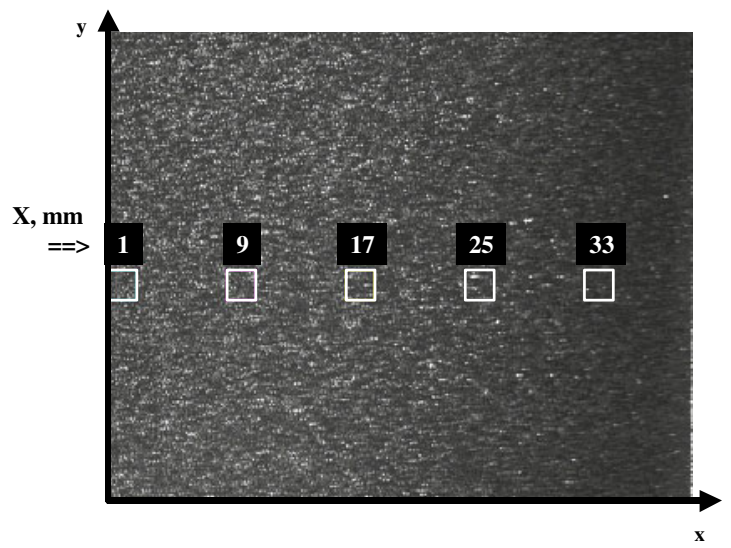

Figure 4. PIV image identifying $64 \times 64$ pixel regions enlarged in figures 5 and 6.

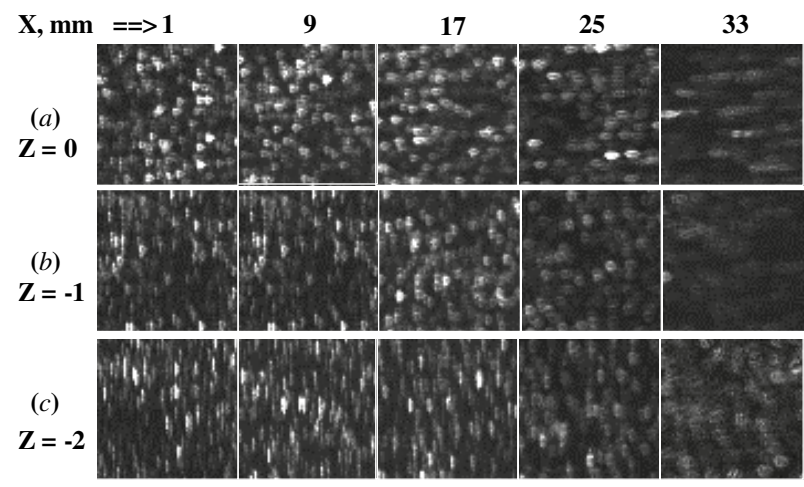

Figure 5. Enlargement of $64 \times 64$ pixel regions in figure 4 showing image aberrations.

\subsection{Image aberrations}

The effect of the cylinder aberrations on particle-image quality can be observed in the arbitrarily chosen PIV image shown in figure 4 , which was recorded at the centre of the cylinder, $(z=0)$. Five $64 \times 64$ pixel subregions of the image in figure 4 have been expanded and are shown in figure 5(a), $z=0$. The numbers indicate the value of $x$ at the centre of the subregions. The sequence shows that the images are approximately round near $x=0$ where the best focus was performed. With increasing $x$, the sagittal rays become more focused and the meridional rays become less focused, resulting in images that are elongated in the $x$ direction. This change with increasing $x$ is consistent with the meridional and sagittal positions illustrated in figure 1 and the measurements presented in figure 3.

Figure 4 also suggests that there is an apparent decrease in particle-number density (number per unit area) or at least a lower intensity moving from $x=0$ to the cylinder wall (large $x$ ); this is in spite of the fact that the particle-number density in the cylinder (number per unit volume) is quite uniform. Figure 5( $a$ ) reveals that this is because particle images at large $x$ have lower intensity than equivalent particles at $x=$ 0 . One might presume that the lowest intensity images are not even recorded at large values of $x$. There are two causes for the lower particle-image intensity $\left(\mathrm{W} \mathrm{mm}^{-2}\right)$. One is defocusing of the scattered light, which creates larger images thereby distributing the light of each particle's image over a larger area 


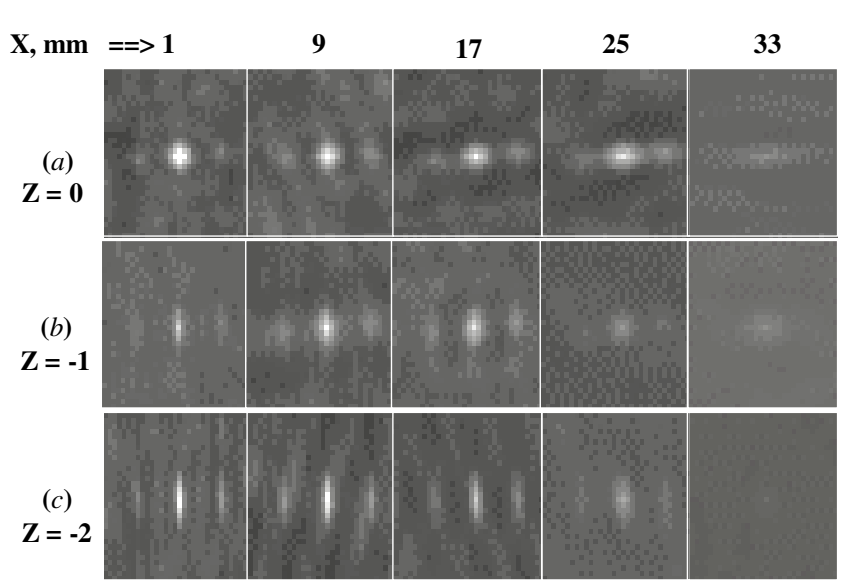

Figure 6. Correlations of the $64 \times 64$ pixel regions in figure 5 .

in the image plane. The second cause of the lower intensity is that less light is transmitted through the cylinder to the camera lens for particles at large values of $x$. This transmission loss occurs because the angle between the scattered rays and the normal to the cylinder wall is larger than those scattered near $x=0$. Consequently, more light is reflected and less transmitted as per Fresnel's equations (see Fowels 1975). Further, since light with transverse electric (TE) polarization transmits less at large angles compared to transverse magnetic (TM), polarization, light from the $y$-polarized laser is less and pairing loss may occur. This pairing loss is only an issue for cross-polarized laser illumination, which is used here to create the electro-optical image shift. This would not be an issue for dual-frame cross-correlation PIV, where both lasers can have the same direction of polarization. This further suggests that it is best to have TM polarization (both lasers' polarizations parallel to the $x-z$ plane), to somewhat improve the particleimage intensity at large values of $x$.

As noted previously, the field curvature results in the focal positions moving in the $-z$ direction with increasing $x$. It is reasonable that moving the camera toward the curvature might locate a compromise position where the images would provide better PIV interrogations. Thus, it is of interest to determine the extent to which the images change as the camera (image plane) is moved toward the object plane ( $-z$ direction). This is shown in figures $5(b)$ and $(c)$ where the camera (image plane) was moved to $z=-1$ and $-2 \mathrm{~mm}$, respectively. At each of these image planes the position of the CLC can be identified as the position where the images appear most round, $(x, z)=(1,0)$, $(25,-1)$ and $(33,-2)$. That is, as $x$ increases the camera need be moved toward the cylinder to focus on the CLC in the image. Also, the size of the CLC image increases as $z$ becomes more negative. Finally, whereas the sagittal focus is approached at $x=33$ for $z=0$, it disappears at $z=-1$ and -2 . Instead, meridional focusing appears for values of $x$ smaller than the CLC as the flat image plane of the camera moves toward the meridional focal plane at $x=0$.

\subsection{Correlations}

Figure 6 shows the correlations for each of the $64 \times 64$ subregions in figure 5. The displacement peaks, located to the right and left of the self-correlation peak, are the correlations of (a)
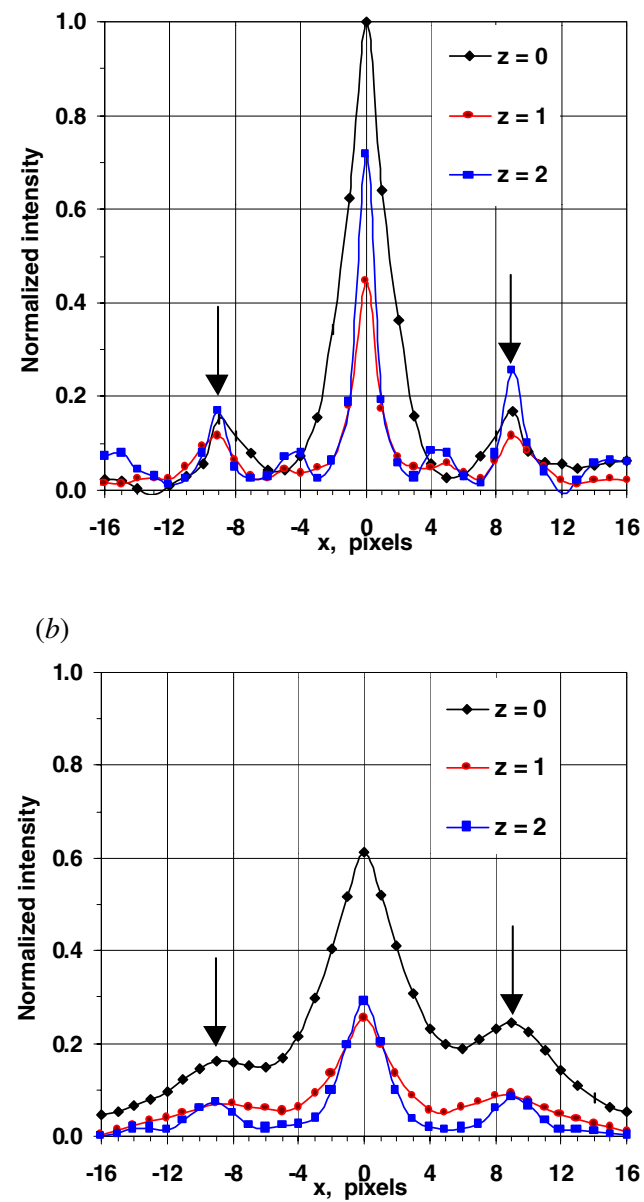

(c)

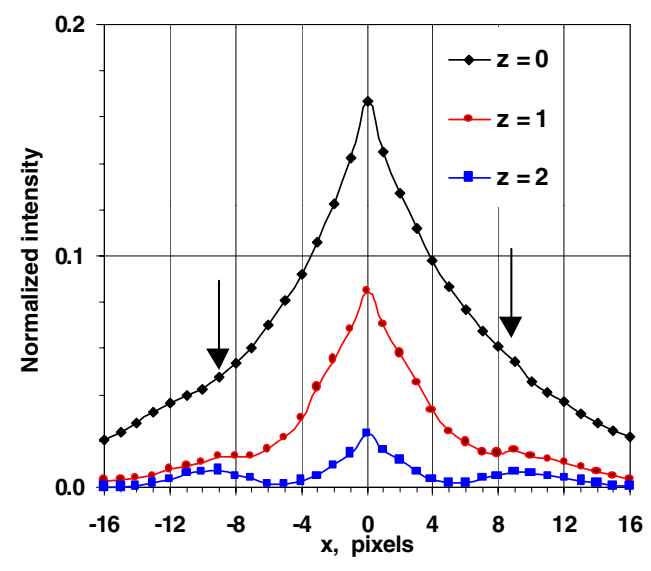

Figure 7. Line scans through the correlations in figure 6. Arrows indicate positions of the displacement-correlation peaks. (a) $x=1 \mathrm{~mm}$, (b) $x=25 \mathrm{~mm}$ and (c) $x=33 \mathrm{~mm}$.

each particle image with its paired image from the other of the two exposures. The shapes of the correlations become oblong, commensurate with the image aberrations. For examples, see the sagittal, CLC and meridional correlations at $(x, z)=$ $(33,0),(1,0)$ and $(0,-2)$ respectively.

The self-correlation peak, located at the centre of each correlation region, provides a measure of the mean particle- 


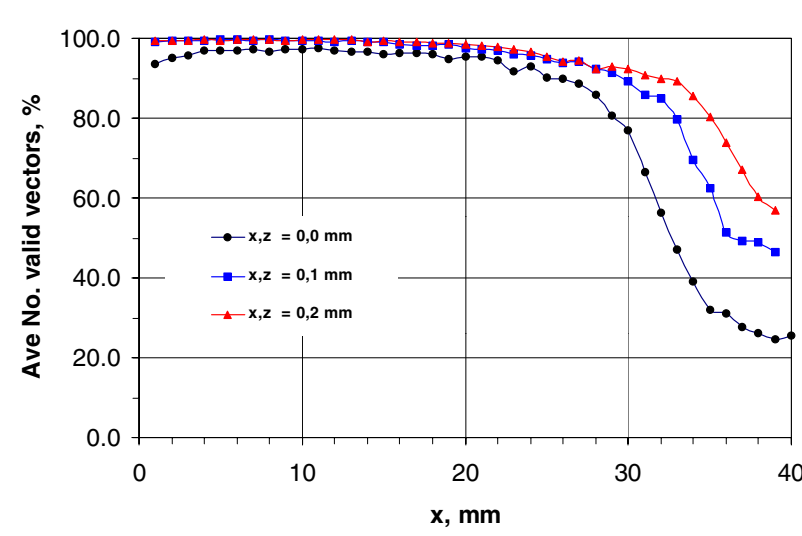

Figure 8. Number of valid displacement peaks detected, a measure of displacement-peak detectability.

image diameter. Following the theory of Westerweel (1997), the correlation diameter at the $e^{-2}$ point is $2^{1 / 2}$ times the mean particle-image diameter. In figure 7 , the amplitude is given for the correlations in figure 6 . It can be observed that the smallest correlation-peak diameter occurred for $x=1$ and $z=0$. This can be expected because $x=1$ is nearly on the cylinder axis where the sagittal and meridional planes are closest. In these images, the correlation diameter for $(x, z)=(1,0)$ at $e^{-2}$ is about 3 pixels, yielding an estimate of the mean particle diameter of $>2$ pixels (cf figure 5). This is slightly larger than the 1.5 pixels measured with the same system but flat windows by Megerle et al (2002). Thus the particle-image diameter, even at the best focus position, is quite large compared to that expected from $1 \mu \mathrm{m}$ particles and $M=0.214$. This is, of course, due to the lens and cylinder aberrations as well as the under-sampling of the digital recording technique (small number of pixels per image). Further, the increased image aberrations at large $x$ and $z$ discussed in section 3.2 impact the displacement correlations for each interrogated region as seen in figures 6 and 7. The aberration of the correlations raises two questions. First, how is the detection of the signal peak affected and, second, how is the precision and accuracy of the displacement affected?

\subsection{Effects on PIV}

The effect of the aberrations on peak detection was evaluated by quantifying the number of valid vectors detected by the PIV interrogation algorithm at each grid position. Here a peak-to-noise ratio of 1.3 was required within \pm 2 pixels of the expected 8.7 pixel displacement. This is plotted in figure 8 as a function of $x$ for the three $z$-image-plane positions in figures 5 and 6 . To generate figure 8 , the number of detected vectors in the ensemble average was determined at each grid point; these were then averaged over the $y$ direction. Two important observations can be made from the data in figure 8 . First, the number of valid vectors decreases near the cylinder wall (increasing $x$ ), due both to the loss of transmitted light and to pairing losses as described previously. Using $90 \%$ successful vector detection as a criterion for useful statistics, the measurements are useful out to $x=26,30$ and $32 \mathrm{~mm}$ for $z=0,-1$ and $-2 \mathrm{~mm}$, respectively. The second important observation is that the number of detected vectors increases as the camera is moved toward the cylinder, which is moving (a)

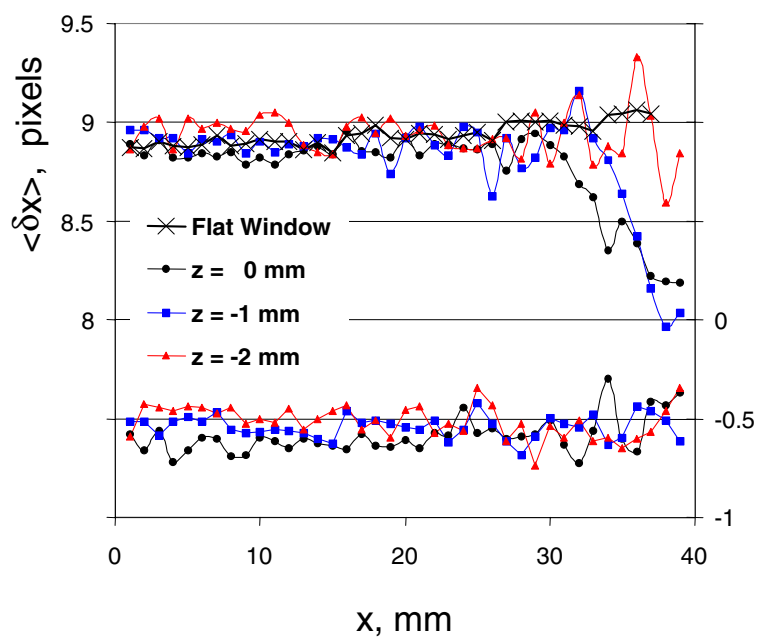

(b)

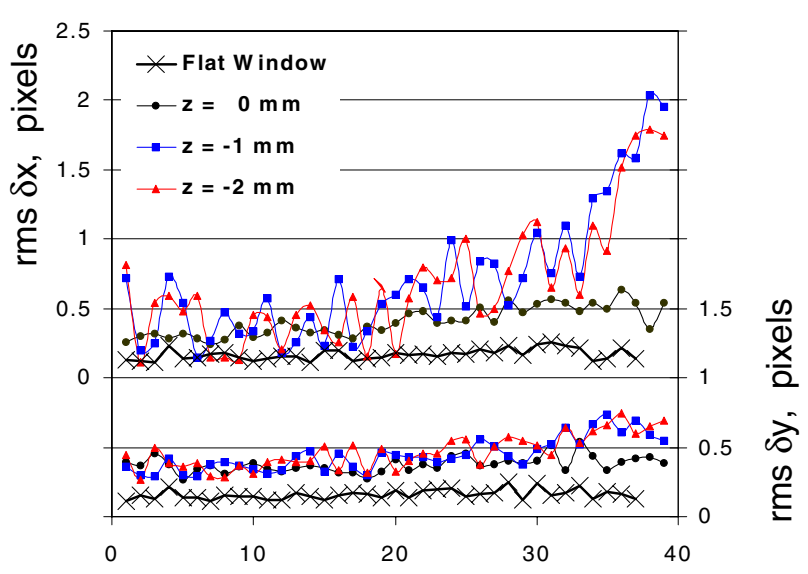

$\mathrm{x}, \mathrm{mm}$

Figure 9. Average $(a)$ and $\mathrm{rms}(b)$ displacements at $y=0$, compared with the flat-window tests of Megerle et al (2002).

the camera's image plane toward the CLC for large $x$. This can be explained by the observed changes in the correlations at large $x$, as shown in figure 7 . First, observe the correlation intensity distribution for $z=0 \mathrm{~mm}$ at $x=1,25$ and $33 \mathrm{~mm}$ in figures $7(a),(b)$ and $(c)$, respectively. The width of the self-correlation peak (pixels) becomes large enough that it is much larger than the 9 pixel displacement of the images (i.e. the image dimension is as large as the displacement). As a result, the self-correlation and displacement peaks become indistinguishable. This is because the meridional rays are out of focus as the sagittal focus is approached, as was observed in figures $5(a)$ and $6(a)$. However, figure $7(c)$ shows that, as the camera was moved toward the cylinder $(z=-1$ and $-2 \mathrm{~mm})$, the width of the self-correlation peak became smaller and the displacement peak became distinct.

The effect of the aberrations on the measurement accuracy was determined by measuring the change in the ensembleaverage displacement, $\langle\delta x\rangle$ and $\langle\delta y\rangle$, both as a function of $x$ and with changes in the camera position, $z$. The results are shown in figure $9(a)$ for $y=0$. The displacement distribution measured when replacing the cylinder with a flat window is shown for comparison (the flat-window data were taken 
from Megerle (2002) for $M=0.214, f / 8$, best focus, and optimal seed density). The data show that $\langle\delta x\rangle$ decreases for $x>30 \mathrm{~mm}$ at $z=0$, causing a $10 \%$ error near the wall. $x=30 \mathrm{~mm}$ is slightly larger than the value of $x=26 \mathrm{~mm}$ where the correlation displacement-peak detection fell below $90 \%$ (cf figure 8 ). When $z$ was increased from 0 to -2 the error in $\langle\delta x\rangle$ decreases (accuracy improves); this is due to the fact that the image plane is now closer to the CLC at $x>30 \mathrm{~mm}$. In contrast, $\langle\delta y\rangle$ appears uniform over the entire region. We presume $\langle\delta y\rangle$ is insensitive, because for $x>25 \mathrm{~mm}$, all three image plane positions, $z$, lie between the sagittal focus and the CLC. Thus, the peaks in the $y$ direction are not significantly broadened. Further, for the electro-optical shift imposed here the self-correlation peak does not interfere with the displacement peak in the $y$ direction, which would not necessarily be true in a random flow.

The effect of the aberrations on the precision of the PIV displacement measurements was determined by measuring $\operatorname{rms}(\delta x)$ and $\operatorname{rms}(\delta y)$ about the ensemble means in figure 9. These data show that the rms noise of both $\delta x$ and $\delta y$ increases for $x>20 \mathrm{~mm}$, although it is much greater for $\delta x$. Further, the noise is lowest for imaging at $z=0$, where the rms noise is between 0.3 and 0.5 pixels for $z=0$. Using the 8.8 pixel displacement as a reasonable maximum for the $32 \times 32$ interrogation spots used here, the rms noise is between 3.5 and $5.5 \%$ of full scale for $z=0$. Contrary to the improvements in the mean displacements and the number of valid vectors, the noise increased to over $20 \%$ for $x>30 \mathrm{~mm}$ as the camera was moved to $z=-2$. This is presumed to be due to poor detectability, since moving toward the CLC should otherwise decrease the aberration. The flat-window data in figure $9(b)$ demonstrate that, at best, the noise when imaging through the cylinder $(z=0)$ is two to three times greater than the noise when imaging through flat windows.

\section{Conclusions}

The results above have identified the two factors that degrade the quality of the particle image when imaging through the cylinder. Namely, aberrations result in poor focusing and low light transmission near the wall. These two factors lead to poor vector detectability and errors in the vectors. Thin walled cylinders (not studied here) would have reduced aberrations but still suffer from low-light transmission at large radii.

The detectability was quantified as the number of valid vectors. For the single-exposure cross-correlation used here, the percentage of valid vectors fell to below $80 \%$ for $x>$ $30 \mathrm{~mm}$; however, this could be improved by moving the camera to $z=-2 \mathrm{~mm}$. This detectability problem was shown to result from the broadening of the self-correlation peak. It is presumed that this could be greatly improved with double-image crosscorrelation.

The accuracy degradation with increasing $x$ was determined by looking at the change in the ensemble average displacement of the 60 images. The average displacement changed after $x=30 \mathrm{~mm}$ and improved by moving the camera to $z=-2 \mathrm{~mm}$

The imprecision was quantified as the rms fluctuations of the displacement about the ensemble average. This noise was found to be $3.5-5.5 \%$ when imaging at $z=0$. Contrary to the previous effects, the imprecision increased dramatically when the camera was moved to $z=-2 \mathrm{~mm}$. The importance of the reduced light transmission at large $x$ is that, even with proper correction of the aberrations, a limiting value of $x$ will occur such that too little scattered light is transmitted. Orienting the lasers for TM polarization will improve this.

Several cautions should be noted concerning these conclusions. First, single-image cross-correlation was used for the interrogations. It is presumed that double-image cross-correlation would improve both the precision and the detectability. The electro-optical image shifting technique used to create a repeatable displacement distribution does not include increased noise and decreased correlation-peak detection due to velocity gradients and pairing losses due to out-of-plane velocity. Finally, due to the laser illumination configuration, these experiments do not include the kind of background noise experienced in operational engine experiments.

\section{Acknowledgment}

This work was supported by General Motors Corporation within the Collaborative Research Laboratory at the University of Michigan.

\section{References}

Adrian R J 1997 Dynamic ranges of velocity and spatial resolution of particle image velocimetry Meas. Sci. Technol. 8 1393-8

Fowels G R 1975 Introduction to Modern Optics 2nd edn (New York: Holt, Rinehart and Wilston)

Keane R D and Adrian R J 1990 Optimization of particle image velocimeters: Part 1. Double pulsed systems Meas. Sci. Technol. 1 1202-15

Landreth C C and Adrian R J 1988 Electrooptical image shifting for particle image velocimetry Appl. Opt. 27 4216-20

Megerle M, Sick V and Reuss D L 2002 Measurement of digital particle image velocimetry precision using electro-optically created particle-image displacements Meas. Sci. Technol. 13 997-1005

Reeves M 1995 Particle image velocimetry applied to internal combustion engine in-cylinder flows PhD Thesis Loughbrough University of Technology, Loughborough, Leicestershire

Reuss D L 1993 Two-dimensional particle-image velocimetry with electrooptical image shifting in an internal combustion engine Proc. SPIE Optical Diagnostics in Fluids and Flows $\mathbf{2 0 0 5}$ 413-24

Reuss D L 2000 Cyclic variability of large-scale turbulence structures in directed and undirected IC engine flows $S A E$ Paper No 2000-01-0246

Westerweel J 1997 Fundamentals of digital particle image velocimetry Meas. Sci. Technol. 8 1379-92 